Journal of the Magnetics Society of Japan Vol. 15 Supplement, No. S2 (1991)

(C) 1991 by The Magnetics Society of Japan

\title{
ANGULAR DEPENDENCE OF THE MAGNETIC PROPERTIES IN RECORDING MEDIA
}

Dennis E. SPELIOTIS and J. Patrick JUDGE

Digital Measurement Systems, Inc., 8 Ray Avenue, Burlington, MA 01803, USA

\begin{abstract}
The writing process in magnetic recording is vectorial, with the head field changing from Iongitudinal to perpendicular as the media moves by the gap of the recording transducer. Therefore, it is important to study the angular dependence of the magnetic properties of the media in order to understand the nature of the writing-demagnetization processes. To correctly compensate for the demagnetization fields in out-of-plane measurements it is necessary to use vector magnetometry and to include all the components of the demagnetizing fields, except that for the perpendicular direction it is possible to obtain accurate corrected values of the remanence coercivity by using a conventional single-axis vSM. Measurements of this parameter for many state-of-the-art recording media are given, revealing a significant problem for longitudinal thin film media.
\end{abstract}

\section{INIRODUCTION}

Magnetic recording media employ thin films or relatively thin particulate coatings, and thus have a preference for their remanent magnetization to lie in the plane of the media. This preference may be further enhanced if the individual easy axes of the particles or crystallites are also in the plane, or may be offset if they are out of the plane. Coupled with this magnetic anisotropy of the media we have the vectorial nature of the recording head field which changes direction from longitudinal to perpendicular as the media move past the gap of the recording transducer. Therefore, the angular dependence of the magnetic properties of the media is of singular importance in understanding the writing-demagnetization processes. since the remanence coercivity $\mathrm{H}_{x}$ represents the actual switching criterion of the media, we concentrated our major emphasis in this study to the determination of the angular variation of this parameter.

The measurement of the angular dependence of the magnetic properties in the plane of the media is quite straight forward, but the out-of-plane measurements are complicated by the presence of the demagnetizing fields. For uniaxial, Iongitudinally oriented media, it has at times been assumed that the magnetic properties are iso- tropic in a plane perpendicular to the orientation axis, but this assumption is not valid for most media, since the distribution of the particle or crystallite anisotropies in the plane orthogonal to the axis of orientation is not uniform.

To compensate for the inherent demagnetizing fields in out-of-plane measurements it is necessary to use vector magnetometry [1-4] in order to determine the magnitude and the angle of the effective field $\mathrm{H}_{e}$. The main complication arises from the fact that as the magnitude of the externally applied field $\mathrm{H}_{\mathrm{a}}$ changes, both the magnitude and the angle of the effective field change. It thus becomes necessary to use euristic iterative techniques [4] and continuously readjust the magnitude and the angle of the applied field so as to vary only the magnitude of the effective field but keep its angle with respect to the sample plane constant. The measurement is quite complicated and lengthy, prompting us to investigate some alternative approximate techniques that may yield useful--even though not exact--results, and to determine the conditions under which these simplified methods for demagnetization corrections may be used instead of the full vectorial approach.

In the following sections we first describe the different simplified models for partially compensating 
the demagnetizing fields. We then present the experimental measurements of the angular dependence of $\mathrm{H}_{x}$ and $\mathrm{H}_{\mathrm{c}}$ in the plane of various longitudinally oriented recording media, followed by out-of-the-plane measurements of $H_{x}$ for longitudinally and perpendicularly oriented particulate and thin film media, along with the corrections for demagnetization based on the simplified models. Finally, we assess the conditions of applicability of the simplified models.

\section{MODETS FOR DEMAGNETIZATION FIELD CORRECTION}

The only way to completely and correctly compensate for the demagnetizing fields is to use vector magnetometry in order to calculate the effective internal field $\mathrm{H}_{-}$acting on the sample when an external field $\mathrm{H}_{\mathrm{a}}$ is applied at some angle with respect to the plane of the sample. As pointed out above, however, this is rather complicated and time consuming, and we would like to explore whether, and under what conditions the following three simplified models in measuring $H_{x}(\Omega)$ may yield useful results.

Referring to Fig. 1, we start by first measuring the uncorrected angular variation of the remanence coercivity $\mathrm{H}_{-}^{\prime}(\Omega)$, with the external fields applied along the $x$-axis, and the sample plane rotated to an angle $\Omega$ with respect to the applied field. In our notation here we use the accent (') to denote uncorrected values of $H_{x}$, the subscripts $d$ and $e$ to designate the demagnetizing and the effective fields, respectively, and the symbols (1) and (/I) to designate directions perpendicular and parallel to the sample plane, respectively.

\section{(A) $M_{2}$ or hard-axis model}

In this simplified model we assume a well-aligned sample with uniaxial anisotropy, and its magnetization reversal proceeds by rotation. It is then reasonable to assume that with $H_{x}^{\prime}$ (R) applied, the average magnetization is along the hard axis of the par-

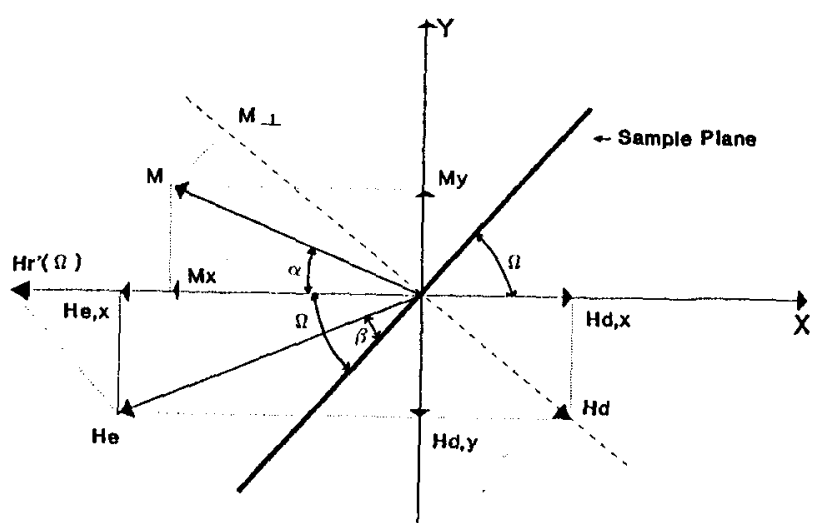

Fig. 1. Vector geometry of sample showing the applied and the demagnetizing fields.

ticles or crystallites, and when $H_{x}^{\prime}(\Omega)$ is reduced to zero, the magnetization rotates back with equal probability along the plus and minus directions of the axis of alignment. The sample would thus exhibit zero remanence along any axis in the plane or out of the plane after the removal of each particular $\mathrm{H}_{r}^{\prime}(\Omega)$. We distinguish two cases:

\section{Easy axis in the sample plane}

With $H_{x}^{\prime}(\Omega)$ applied we only have $M_{1}$, and the component $M x$ along the field axis that would be measured by a conventional single-axis VSM is:

$M_{x}=M_{\perp} \sin \Omega$

Therefore if we neglect the (1) component of the demagnetizing field $\mathrm{H}_{\mathrm{a}, \mathrm{y}}$ (in order to simplify the problem) we obtain for the corrected value of the remanence coercivity:

$\mathrm{H}_{x}(\Omega)=\mathrm{H}_{x}^{\prime}(\Omega)-4 \pi \mathrm{M}_{x}$

\section{Easy axis 1 to sample plane}

In this case, with $H_{x}^{\prime}(\Omega)$ applied we only have $M / /$, and

$M_{x}=M / / \cos \Omega$

since there are no demagnetizing

fields,

$\mathrm{H}_{x}(\Omega)=\mathrm{H}_{x}^{\prime}(\Omega)$

\section{(B) $M_{x}$ Model}

With $H_{x}^{\prime}(\Omega)$ applied, we assume that $M_{\times}$is the only significant component of the magnetization. Thus, $\mathrm{M}_{\perp}=\mathrm{M}_{\mathrm{x}} \sin \Omega$ 
By again neglecting $H_{d, y}$ in order to simplify the problem we obtain:

$$
\mathrm{H}_{x}(\Omega)=\mathrm{H}_{x}^{\prime}(\Omega)-4 \pi \mathrm{M}_{x} \sin 2 \Omega
$$

\section{(C) Partial vector model}

The two simple models (a) and (b) above require only a conventional uniaxial VSM. For this and the following model, we require, a biaxial (vector) VSM $[1,2]$. With $H_{x}^{\prime}(\Omega)$ applied we measure $M x$ and $M y$, compute the vector $\vec{M}=M \angle \alpha$, where

$M=\left(M_{x}^{2}+M_{y}^{2}\right)^{1 / 2}$ and $\alpha=\tan -1\left(M_{y} / M_{x}\right)$, and obtain

$$
M_{L}=M \cos (90-\Omega-\alpha)
$$

If we again neglect $H_{a, y}$ for simplicity, we finally compute the corrected remanence, coercivity

$$
\mathrm{H}_{x}(\Omega)=\mathrm{H}_{x}^{\prime}(\Omega)-4 \pi \mathrm{M}_{\perp} \sin \Omega
$$

\section{(D) Full vector model}

In all three simplified models above we assumed that we can neglect the $y$-component of the demagnetizing field Ha,y, but in general this assumption is not justified [4]. Therefore for the general case we must determine the effective field $\mathrm{H}_{\mathrm{b}}$ and the angle $\beta$ it makes with the sample plane. Unfortunately, both $H_{0}$ and $\beta$ vary as the applied field changes in magnitude, thus requiring laborious trial and error measurements for the correct determination of $H_{x}(\Omega)$. This will form the subject of a future publication.

\section{EXPERTMENTAL RESULTS AND DISCUSSION}

\section{Angular Measurements in the Plane}

We first show the experimental results of the angular dependence of $\mathrm{H}_{c}$ and $\mathrm{H}_{x}$ in the plane of different recording media with in-plane anisotropy (perpendicular and isotropic media, of course, have no in-plane angular variations of their magnetic properties). The results are shown in Figs. 2-7 for the most common types of media. Co-gamma (SVHS), metal particle $(8 \mathrm{~mm})$, and $\mathrm{Ba}$-ferrite experimental longitudinally oriented (not very well) tape show only a small increase in $\mathrm{H}_{x}$ at 900 , while $\mathrm{CrO}_{2}$ tape shows a

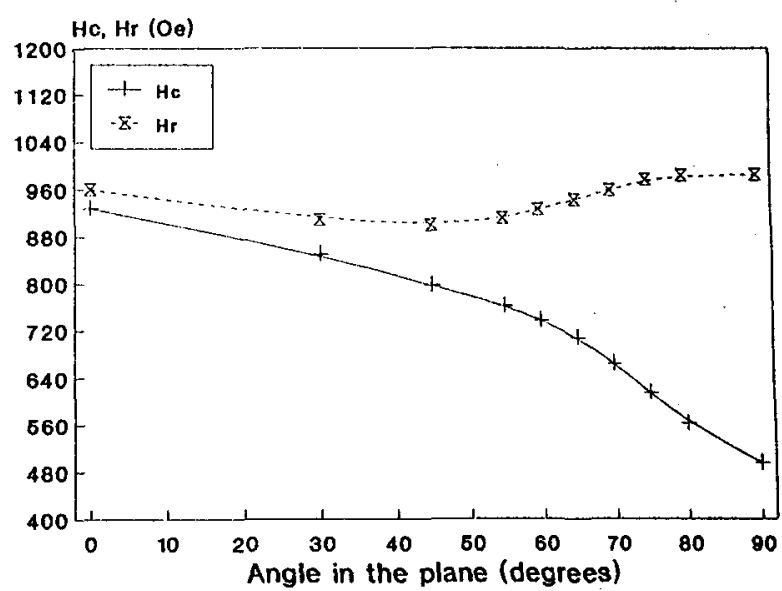

Fig. 2. In-plane angular dependence of $\mathrm{H}_{\mathrm{C}}$ and $\mathrm{H}_{\mathrm{r}}$ for Co-gamma (SVHS) tape.

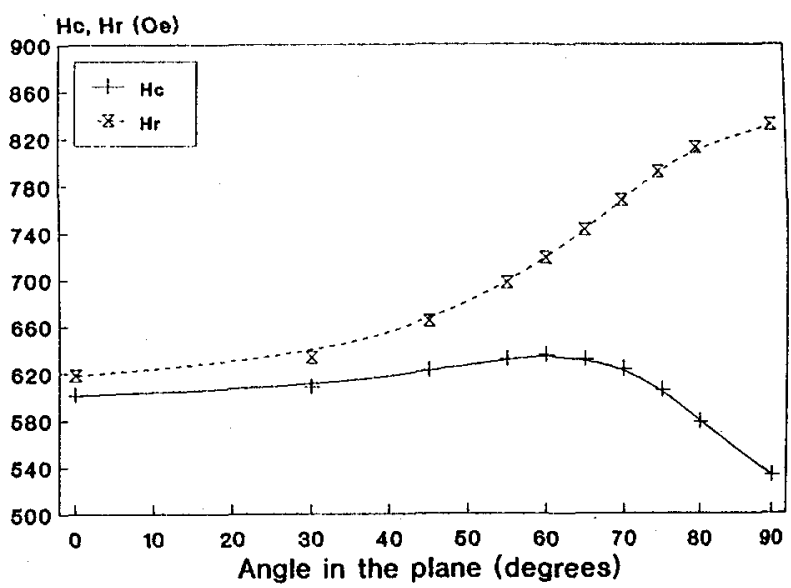

Fig. 3. In-plane angular dependence of $\mathrm{H}_{\mathrm{C}}$ and $\mathrm{H}_{r}$ for an experimental $\mathrm{CrO}_{2}$ tape.

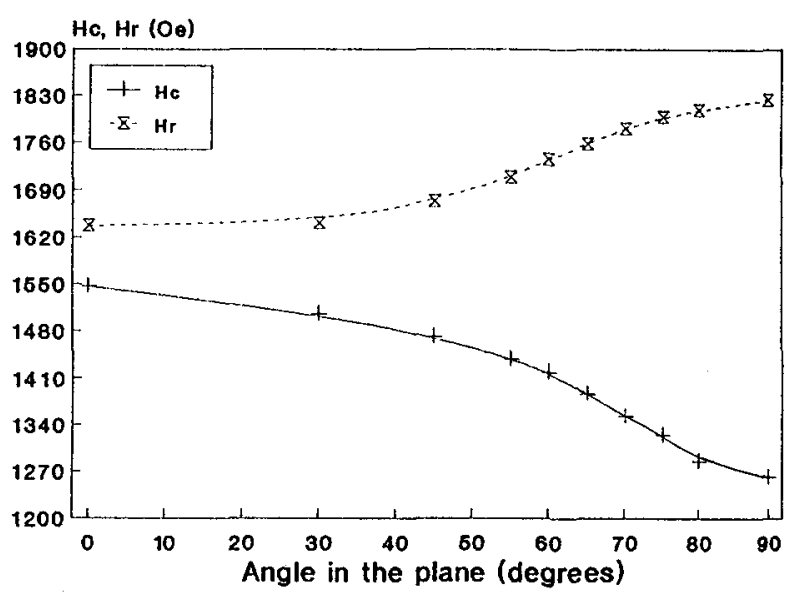

Fig. 4. In-plane angular dependence of $\mathrm{H}_{\mathrm{C}}$ and $\mathrm{H}_{\mathrm{r}}$ for $\mathrm{Hi} 8 \mathrm{~mm} \mathrm{MP}$ tape. 


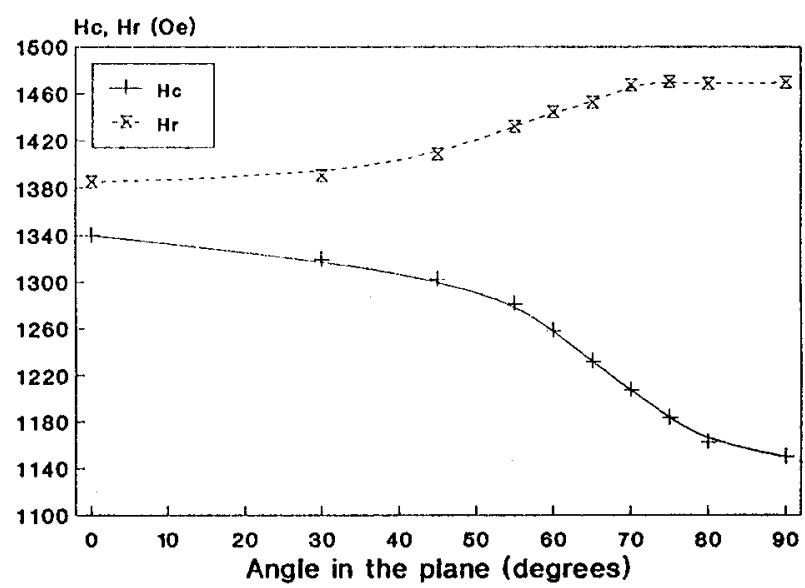

Fig. 5. In-plane angular dependence of $\mathrm{H}_{\mathrm{C}}$ and $\mathrm{H}_{\mathrm{r}}$ for an experimental BaFe longitudinal tape.

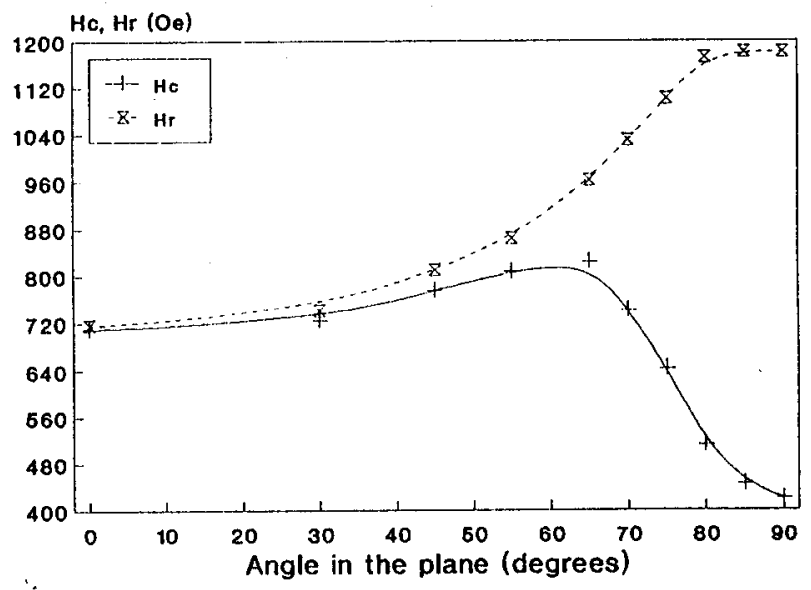

Fig. 6. In-plane angular dependence of $\mathrm{H}_{C}$ and $\mathrm{H}_{\mathrm{r}}$ for longitudinal BaFe rigid disk.

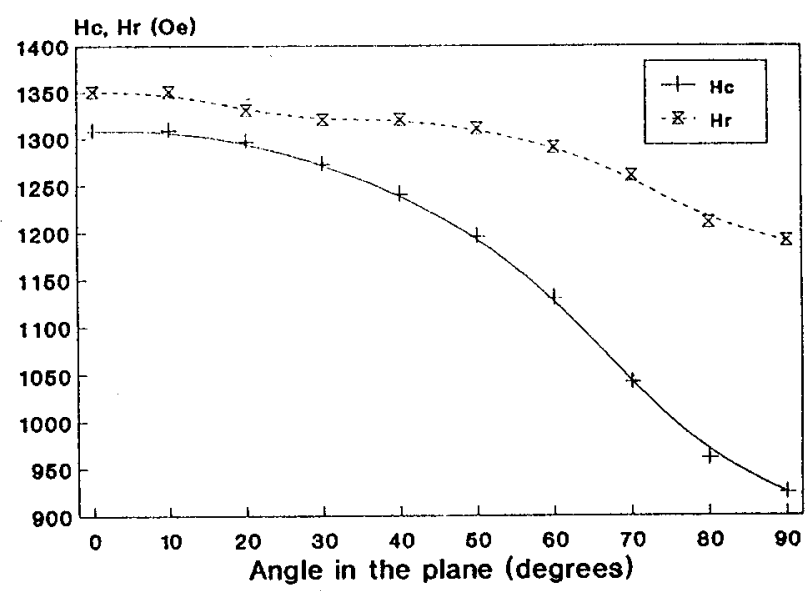

Fig. 7. In-plane angular dependence of $\mathrm{H}_{\mathrm{C}}$ and $H_{r}$ for anisotropic thin film rigid disk。 large increase, and Ba-ferrite rigid disk media show a very large increase. The Ba-ferrite rigid disk media have much better orientation compared with the Ba-ferrite tapes, and this accounts for the much larger $\mathrm{DH}_{\mathbf{r}}$ of the former, where $\mathrm{DH}_{x}$ is defined by:

$$
\mathrm{DH}_{x}=\left[\mathrm{H}_{x}(900)-\mathrm{H}_{x}(00)\right] / \mathrm{H}_{x}(00)
$$

In sharp contrast to the particulate media, anisotropic rigid disk sputtered thin film media show a substantial drop in $H_{x}$ from the circumferential to the radial directions.

\section{Angular measurements out of the plane}

We measured the out of plane angular variation of the remanence, coercivity of various particulate media, and applied the approximate demagnetization corrections according to models A, B, and C described in the previous section. All the models agree with each other as well as with the full vector model $D$ at 00 and at $90^{\circ}$ (perpendicular direction). For other angles, the demagnetization corrections provided by the three simplified models vary over a fairly wide range, depending on the type of media [3]. Of the three simplified models, we might expect that model $C$ may provide the best approximations for the corrected $\mathrm{H}_{2}$ values, and the best criterion to use in assessing how good these approximations are is how small is the angle $(\Omega-\beta)$ between the applied field $\mathrm{H}_{r}^{\prime}(\Omega)$ and the effective field $\mathrm{H}_{-}$; the smaller the angle, the better the approximation. Depending on the type of media and the direction of magnetic orientation we can make the following statements:

(a) Longitudinally oriented particulate media

These include Co-ganma, $\mathrm{CrO}_{2}$, Metal Particle $8 \mathrm{~mm}$ (MP), and experimental Ba-ferrite tapes. The angular deviation between $H_{\infty}$ and $H_{r}$ is fairly small (up to $12^{\circ}$ maximum). Correction models $B$ and $C$ agree quite well. 
(b) Isotropic particulate media

These include $4 \mathrm{MB}$ Ba-ferrite floppy disk media and random in-plane MP floppy disk media. The angular deviation between $\mathrm{H}_{e}$ and $\mathrm{H}_{x}$ is larger than in (a) above (up to about $20^{\circ}$ maximum), but the corrections provided by models $\mathrm{B}$ and $\mathrm{C}$ are almost identical.

\section{(c) Perpendicularly oriented media}

These include experimental Ba-ferrite particulate and sputtered $\mathrm{CoCr}$ thin film media. The angular deviation between $\mathrm{H}_{e}$ and $\mathrm{H}_{x}$ is quite small (up to $50 \max$ ), but the agreement between correction models $B$ and $C$ is not very good.

\section{(d) Thin film longitudinal media}

These include the typical high coercivity sputtered thin film media used for small rigid disks, including CocrTa and CoNicr compositions. Here, the angular deviation between $\mathrm{H}_{6}$ and $\mathrm{H}_{x}$ is very large, and only the full vector correction (Model D) can be used for angles other than $0^{\circ}$ and $90^{\circ}$.

In Table 1 we list the values of the remanence coercivities in the longitudinal and perpendicular directions for a representative collection of typical high density recording media (both commercial and experimental), and also the computed $\mathrm{DH}_{x}$ values.

The co-modified iron oxide media (COFe) used for SVHS video show a small positive $\mathrm{DH}_{x}$, even though they have excellent longitudinal orientation. The $\mathrm{CrO}_{2}$ and MP tapes have large positive $\mathrm{DH}_{r}$ values. The $\mathrm{Ba}-$ ferrite longitudinally oriented experimental tape was not very well oriented (longitudinal $S Q=0.67$ ) because a good percentage of the Ba-ferrite platelets are apparently tilted towards the surface of the substrate film, thus resulting in a relatively small $\mathrm{DH}_{r}$ value. If this tilting of the Ba-ferrite platelets can be avoided, the $\mathrm{DH}_{\mathrm{x}}$ values would increase dramatically, and would surpass those of any other medium. The isotropic $\mathrm{Ba}-$ ferrite floppy disk sample shows a negative $\mathrm{DH}_{x}$, because it is not really
Table 1

Renanence coercivity Variation of Different Roconding Hedia

\begin{tabular}{|c|c|c|c|}
\hline Recording Mediu & $\underset{0 e}{\mathbb{H}_{r}(1 /)}$ & $\mathrm{H}_{5}(\mathrm{~d})$ & $\begin{array}{l}\mathrm{DA}_{2} \\
(\mathrm{z})\end{array}$ \\
\hline $\operatorname{cog}_{e}(\mathrm{SVES})$ & 942 & 1006 & +6.8 \\
\hline $\mathrm{CrO}_{3}(\mathrm{Brptl})$. & 596 & 800 & +34.2 \\
\hline $\begin{array}{l}\operatorname{lp}(8 x a) \\
(y)\end{array}$ & 1544 & 2230 & +41.4 \\
\hline $\begin{array}{l}\text { Bape(Exptl.) } \\
\text { (II) }\end{array}$ & 1344 & 1460 & +8.6 \\
\hline $\begin{array}{l}\text { Bafe (4NB) } \\
\text { (Isotropic) }\end{array}$ & 734 & 627 & -14.6 \\
\hline $\begin{array}{l}\text { Bafe(Exptl) } \\
(1) \\
\operatorname{CoCr}(\text { Exptl) }\end{array}$ & 1790 & 1274 & +40.5 \\
\hline$\frac{(1)}{\text { Thin Filn } \mathrm{s}}$ & 2169 & 1616 & +34.2 \\
\hline (II) & 1332 & 730 & -45.2 \\
\hline (III) & 1451 & 987 & -32.0 \\
\hline
\end{tabular}

isotropic. Apparently, a large number of the platelets tend to tilt towards the substrate.

The perpendicularly oriented media (both the Ba-ferrite particulate and the $\mathrm{CoCr}$ sputtered thin film types) show a large increase in $H_{x}$ from the easy axis ( $\perp$ ) to the hard axis $(/ /)$.

The most serious problem occurs in the sputtered longitudinal thin film media used for rigid disk applications. In Table 1 we show two examples of such media: Film A with high in-plane circumferential orientation, and Film $B$ which is isotropic in the plane. Both of these thin film media show large negative $\mathrm{DH}_{x}$ values.

The implications of the angular variation of $H_{x}$ out of the plane are very important with respect to recording demagnetization, while the angular variation of $\mathrm{H}_{x}$ in the plane directly affects side-track writing at high track densities. To reduce or eliminate the effects of these problems it is very desirable to have large posi- 
tive $\mathrm{DH}_{x}$ values in the perpendicular and also in the transverse directions. our findings show that longitudinal thin film media face a serious problem with respect to these considerations, since their remanence coercivities tend to decrease significantly as the angle is changed from the easy to the hard directions of the media.

\section{CONCLUSIONS}

The angular dependence of the remanence coercivity of magnetic recording media has a direct and significant effect on their recording performance. The variation of $H_{x}$ in the plane affects side-track writing, while the variation of $H_{x}$ out of the plane affects recording demagnetization.

In this study we measured the in-plane variation of $H_{x}$ for various state of the art anisotropic media, and found a very large increase for well-oriented $\mathrm{Ba}$-ferrite and $\mathrm{CrO}_{2}$ media, and a moderate increase for MP and Co-gamma tape media. Sputtered longitudinal thin film media exhibit a negative $\mathrm{DH}_{x}$ in the plane, making them more susceptible to side-track writing.

We also measured the out of plane angular variation of $H_{x}$ and used different simplified models to compensate for the demagnetizing fields. Again we found a serious problem in the longitudinal thin film media, where their large negative $\mathrm{DH}_{x}$ values render them vulnerable to recording demagnetization effects. The correct compensation for demagnetizing fields in out-of-plane measurements requires the use of vector magnetometry with full consideration for all the components of the demagnetizing field.

\section{REFERENCES}

[1]. K. Ouchi and S. Iwasaki, IEEE Trans. Magn., vol. 24, pp. 3009-3011, November 1988.

[2]. D. Speliotis, D. Bono, and P. Judge, J. Magn. Soc. Japan, vol. 13, supplement No. S1, 1989.

[3]. D.E. Speliotis and J. P. Judge, paper EB-11 presented at the joint MM/Intermag conference, Pittsburgh, PA, June 1991.

[4]. J.P.C. Bernards and H.A.J. Cramer, paper $C E-15$ presented at the joint MMM/Intermag Conference, Pittsburgh, PA, June 1991.

[5]. H.N. Bertram, IEEE Trans. Magn., vol. 20, pp. 2094-2104, November 1984 .

[6]. D.E. Speliotis, J. Appl. Phys., vol. 63, pp 3432-3434, April 1988. 